\title{
Influência da Umidade na Resistência da Madeira de Eucalipto a Impactos
}

\author{
Anderson Pertuzzatti ${ }^{1}$, Bruno Conte ${ }^{1}$, André Luiz Missio ${ }^{1}$, Darci Alberto Gatto ${ }^{2}$, \\ Clovis Roberto Haselein ${ }^{1}$, Elio José Santini ${ }^{1}$ \\ ${ }^{1}$ Centro de Desenvolvimento Tecnológico, Universidade Federal de Pelotas - UFPel, Pelotas/RS, Brasil \\ ${ }^{2}$ Laboratório de Produtos Florestais, Departamento de Ciências Florestais, Universidade Federal de Santa Maria - UFSM, \\ Santa Maria/RS, Brasil
}

\section{RESUMO}

O estudo teve como objetivo avaliar a resistência da madeira de Eucalyptus grandis e Eucalyptus cloeziana submetidas a diferentes condições de umidade a impactos. Para tanto, os corpos de prova foram distribuídos em três condições de umidade (seco, climatizado e saturado). Foram avaliadas as propriedade de resistência a impactos, massa específica e teor de umidade de equilíbrio. Os dados foram interpretados por meio de análise de variância fatorial. Dessa maneira, verificou-se que a madeira de E. cloeziana foi mais resistente a impactos na condição saturada em comparação com as amostras climatizada e seca. Já o $E$. grandis não apresentou diferença significativa entre as condições de umidade testadas. O E. cloeziana demonstrou maior capacidade de absorver impactos em relação ao E. grandis. Ambas as espécies foram classificadas como pouco resistentes a impactos. Portanto, não é recomendado a utilização das espécies estudadas em locais sujeitos a cargas de choque.

Palavras-chave: flexão dinâmica, qualidade da madeira, Eucalyptus grandis, Eucalyptus cloeziana.

\section{The Influence of Moisture in Bending Strength of Eucalypt Wood}

\begin{abstract}
The study aimed to evaluate the dynamic bending strength of Eucalyptus grandis and Eucalyptus cloeziana woods when subject to different moisture conditions. Samples were divided into three moisture conditions (dry, air-conditioned and wet). The dynamic bending properties, density and equilibrium moisture content were evaluated. The collected data were analyzed through factorial analysis of variance. E. cloeziana wood in the wet condition was more resistant than the dried and air-conditioned samples. On the other hand, dynamic bending strength of E. grandis wood was not significant for the moisture conditions studied. E. cloeziana wood showed higher ability to absorb the impact than E. grandis wood. Both species were classified as low bending strength. Therefore, the use of these species should not be recommended in places with shock loads.
\end{abstract}

Keywords: dynamic bending, wood quality, Eucalyptus grandis, Eucalyptus cloeziana. 


\section{INTRODUÇÃO}

O gênero Eucalyptus expandiu-se rapidamente no cenário brasileiro em virtude do seu elevado potencial produtivo, com madeira de boa aparência e características físicas e mecânicas desejáveis, que o potencializaram como matéria-prima alternativa para o mercado madeireiro (Gonçalez et al., 2006). Em 2014, o Brasil detinha uma área plantada de 5,56 milhões de hectares de eucalipto, gênero florestal mais cultivado no país (IBA, 2015).

Com o alto interesse econômico na madeira desse gênero, encontram-se diversas pesquisas voltadas à caracterização das propriedades físico-mecânicas do material, que se direcionam ao aproveitamento do produto dentro do setor industrial madeireiro. No entanto, muitas vezes não são computadas em ensaios que visam a caracterização da madeira situações diferenciadas resultantes, por exemplo da interface do meio físico com o mecânico.

Em geral, as propriedades mecânicas da madeira aumentam com o decréscimo do teor de umidade (Bodig \& Jayne, 1993; Kretschmann, 2008), o que se explica pelo aumento do adensamento e da rigidez das paredes celulares, bem como da porcentagem de material lenhoso, causado pela perda de água e contração da madeira (Echenique \& Robles, 1993). No entanto, essa condição não se aplica para os testes de flexão dinâmica, pois não apresenta tendência definida. Estudos realizados por Logsdon \& Calil (2002) verificaram que existe uma alta relação entre resistência mecânica e umidade na madeira, que resulta em resistência praticamente constante acima do ponto de saturação das fibras (PSF). Contrariamente, abaixo do PSF, a resistência do material aumenta conforme o teor de umidade diminui.

O conhecimento do ensaio de flexão dinâmica está ligado diretamente à propriedade de resistência a impactos a que a madeira é submetida, sendo importante tanto para fins de segurança como para economicidade (Beltrame et al., 2012). A madeira, quando utilizada para fins estruturais, expõe-se a diferentes condições de umidade relativa, que causam absorção e adsorção de água, as quais interferem diretamente na variação dimensional (Batista \& Klitzke, 2010).

Dessa forma, o presente estudo objetivou avaliar a resistência a impactos da madeira de Eucalyptus grandis W. Hill ex. Maiden e Eucalyptus cloeziana F. Muell. quando submetida a diferentes condições de umidade.

\section{MATERIAL E MÉTODOS}

Para a realização do experimento foram amostradas ao acaso 12 árvores, com 21 anos de idade, sendo 6 de Eucalyptus grandis e 6 de Eucalyptus cloeziana, procedentes de um plantio homogêneo da região central do Rio Grande do Sul (29 $43^{\prime} 01^{\prime \prime}$, 53 $53^{\circ} 43^{\prime} 46^{\prime \prime} \mathrm{N}$, 120 metros de altitude). O material foi amostrado conforme a norma da American Society for Testing and Materials D5536-94 (ASTM, 2010), evitando-se indivíduos de bordadura, fuste tortuoso, com presença de bifurcação e diâmetro a altura do peito (1,30 m - DAP) inferior a $25 \mathrm{~cm}$.

Para cada árvore amostrada retirou-se uma primeira tora de $3 \mathrm{~m}$, da qual se confeccionou um pranchão central de aproximadamente $80 \mathrm{~mm}$ de espessura, com a medula incluída e anéis de crescimento orientados. A espessura foi reduzida para $60 \mathrm{~mm}$ com auxílio de uma plaina desengrossadeira e, posteriormente, foram confeccionados 120 corpos de prova nas dimensões $20 \mathrm{~mm} \times 20 \mathrm{~mm} \times 280 \mathrm{~mm}$ (radial, tangencial e longitudinal) para a realização do ensaio de flexão dinâmica em ambas as espécies.

Inicialmente, como padronização, os corpos de prova foram submersos em água por um período de três meses, em seguida foram distribuídos aleatoriamente em três condições de umidade para cada espécie, definindo-se os tratamentos (Tabela 1).

Condição seca: os corpos de prova foram secos em estufa laboratorial com circulação de ar a $103^{\circ} \mathrm{C}$ $\pm 1^{\circ} \mathrm{C}$ até atingirem massa constante.

Condição climatizada: os corpos de prova foram mantidos em câmara climatizada com temperatura ambiente de $20{ }^{\circ} \mathrm{C} \pm 2{ }^{\circ} \mathrm{C}$ e $65 \% \pm 5 \%$ de umidade relativa até atingirem massa constante. Para tal foram feitas medições periódicas da massa dos corpos de prova até o momento em que a variação foi menor que 0,5\% entre uma medição e outra, no período de 24 .

Condição saturada: os corpos de prova foram mantidos submersos em água até a realização dos ensaios de flexão dinâmica.

Corpos de prova de todos os tratamentos foram pesados em balança analítica com precisão de $0,01 \mathrm{~g}$ e mensurados com o uso de paquímetro digital com precisão de $0,01 \mathrm{~mm}$ para a determinação do volume (método estereométrico) e da massa específica aparente, 
Tabela 1. Tratamentos avaliados no estudo.

Table 1. Treatments evaluated in the study.

\begin{tabular}{cccc}
\hline Tratamento & Espécie & Condição de umidade & Número de amostras \\
\hline 1 & & Seco em estufa & 20 \\
2 & E. cloeziana & Climatizada & 20 \\
3 & & Saturada & 20 \\
4 & & Seco em estufa & 20 \\
\hline 5 & E. grandis & Climatizada & 20 \\
\hline 6 & & Saturada & 20 \\
\hline
\end{tabular}

conforme a norma da Associação Brasileira de Normas Técnicas - NBR 7190 (ABNT, 1997).

Para a realização da propriedade de resistência máxima ao impacto foi utilizado o pêndulo de Charpy com capacidade de 100 Joules. Como padronização, os corpos de prova foram impactados no sentido radial, em que cada corpo de prova foi alocado no vão da máquina, igual a $24,0 \mathrm{~cm}$, sendo atingido na região central por um pêndulo que situa-se a 1 metro de altura. Dessa forma, obteve-se o trabalho absorvido (W), em joules, constatado na leitura da escala graduada.

Com os resultados de $\mathrm{W}$ foi possível calcular a resistência ao impacto à flexão $\left(\mathrm{f}_{\mathrm{bw}}\right)$, por meio da normativa da Associação Brasileira de Normas Técnicas - NBR 7190 (ABNT, 1997), através da Equação 1.

fbw $=1000 . \mathrm{W} / \mathrm{b} . \mathrm{h}$

em que: $\mathrm{f}_{\mathrm{bw}}=$ razão entre a energia necessária à fratura do corpo de prova e a área da seção transversal (KJ.m²-2); $\mathrm{W}=$ energia necessária para fratura do corpo de prova $(\mathrm{J})$; b e $\mathrm{h}=$ dimensões nas seções transversal e radial $(\mathrm{mm})$

Após a realização do ensaio de flexão dinâmica retiraram-se amostras laterais de $50 \mathrm{~mm}$ de comprimento de todos os corpos de prova. Em seguida, foram pesadas e colocadas em estufa a $103^{\circ} \mathrm{C} \pm 1{ }^{\circ} \mathrm{C}$ até atingirem massa constante. De posse desses valores, calculou-se o teor de umidade exato dos corpos de prova para as três condições testadas, por meio da Equação 2.

$$
\mathrm{TU}=\left(\mathrm{M}-\mathrm{M}_{0} / \mathrm{M}_{0}\right) \cdot 100
$$

em que: $\mathrm{TU}=$ teor de umidade de base seca (\%); $\mathrm{M}=$ massa do corpo de prova na condição de umidade (g); $\mathrm{M}_{0}=$ massa seca a $0 \%$ de umidade (g).

A análise estatística procedeu-se com delineamento inteiramente casualizado, com duas espécies (E. grandis e E. cloeziana), três condições de umidade (seca, climatizada e saturada) e 20 repetições (corpos de prova). Os dados foram interpretados por meio de análise de variância (ANOVA) fatorial. Primeiramente foram verificadas a normalidade e homogeneidade de variância dos dados, pressupostos antes de qualquer teste paramétrico. Na ANOVA fatorial foram realizadas comparações de médias para os fatores espécie e condições de umidade, pelo teste $\mathrm{F}$ em $5 \%$ de probabilidade de erro. A partir dos resultados, se constatada interação entre os fatores, realizou-se uma ANOVA simples com desmembramento em $5 \%$ de probabilidade de erro e, em caso de rejeição da hipótese nula, foi realizado teste de médias LSD de Fisher, em 5\% de probabilidade de erro.

\section{RESULTADOS E DISCUSSÃO}

Para os corpos de prova testados nas três condições de umidade (seca, climatizada, saturada) verificou-se que para as duas espécies os teores de umidade apresentaram diferença significativa (Tabela 2). Destaca-se que, na condição saturada, o teor de umidade da madeira de E. grandis foi $88 \%$ superior ao de E. cloeziana, fato já esperado devido à menor massa específica da primeira espécie e, consequentemente, maior permeabilidade.

Por meio da análise fatorial, verificou-se que a interação entre os fatores (espécie x TU) foi estatisticamente significativa $(\mathrm{p}<0,05)$ para todas as propriedades avaliadas, isto é, os fatores agem de modo dependente sobre as propriedades analisadas. Já os fatores espécie e teor de umidade, com exceção do TU, para a $\mathrm{f}_{\mathrm{bw}}$, foram estatisticamente significativos $(\mathrm{p}<0,05)$ para todas as propriedades analisadas no estudo (Tabela 3 ).

A massa específica aparente $\left(\rho_{\text {ap }}\right)$ apresentou diferença estatisticamente significativa entre as espécies, mostrando-se $64 \%, 52 \%$ e $21 \%$ superior para o E. cloeziana quando comparadas com as de E. grandis nas condições seca, 
Tabela 2. Teor de umidade para a madeira de E. grandis e de E. cloeziana em diferentes condições de equilíbrio. Table 2. Moisture content of E. grandis and E. cloeziana woods at different equilibrium conditions.

\begin{tabular}{cccc} 
Condição de equilíbrio & E. grandis & E. cloeziana & F \\
\hline \multirow{2}{*}{ Seca } & $3,04 \mathrm{Aa}$ & $3,68 \mathrm{Ba}$ & $38,7^{* *}$ \\
& $-0,24$ & $-0,37$ & $292,5^{* *}$ \\
\hline Climatizada & $13,62 \mathrm{Ab}$ & $14,91 \mathrm{Bb}$ & $300,7^{* *}$ \\
\hline Saturada & $-0,18$ & $-0,28$ & \\
\hline $\mathrm{F}^{*}$ & $151,81 \mathrm{Ac}$ & $-4,69$ & $4630,44^{* *}$ \\
\hline
\end{tabular}

$\mathrm{F}=$ valor de $\mathrm{F}$ calculado na linha (entre espécies); $\mathrm{F}^{*}$ = valor de $\mathrm{F}$ calculado na coluna (na espécie); médias seguidas da mesma letra não diferem estatisticamente entre si, minúscula na coluna e maiúscula na linha, em $5 \%$ de probabilidade de erro (Fisher Test, p > 0,05); ${ }^{* *}=$ significativo em $1 \%$ de probabilidade de erro; valores entre parênteses indicam erro padrão.

Tabela 3. Análise de variância fatorial das propriedades físico-mecânicas da madeira das espécies E. grandis e E. cloeziana em diferentes condições de umidade.

Table 3. Factorial analysis of variance of both physical and mechanical properties of E. grandis and E. cloeziana species at different moisture conditions.

\begin{tabular}{|c|c|c|c|c|c|}
\hline & Fonte & SQ & GL & QM & $\mathbf{F}$ \\
\hline \multirow{4}{*}{$\rho_{\text {ap }}$} & Espécie & 1,77 & 1 & 1,767 & $984,41^{* *}$ \\
\hline & TU & 6,82 & 2 & 3,414 & $1906,74^{\star *}$ \\
\hline & Espécie x TU & 0,179 & 2 & 0,0895 & $50^{\star *}$ \\
\hline & Resíduos & 0,396 & 123 & 0,0032 & \\
\hline \multirow{4}{*}{ W } & Espécie & 7781 & 1 & 7781 & $193,61^{\star *}$ \\
\hline & TU & 641,9 & 2 & 321 & $7,99^{* *}$ \\
\hline & Espécie x TU & 783,69 & 2 & 391,8 & $9,75^{\star *}$ \\
\hline & Resíduos & 4862,9 & 121 & 40,19 & \\
\hline \multirow{4}{*}{$f_{b w}$} & Espécie & 44015,5 & 1 & 44015,5 & $172,6^{* *}$ \\
\hline & TU & 1070,5 & 2 & 535,28 & $2,1^{\mathrm{ns}}$ \\
\hline & Espécie x TU & 2364,2 & 2 & 1182,1 & $4,63^{*}$ \\
\hline & Resíduos & 28881,2 & 113 & 255,1 & \\
\hline
\end{tabular}

$\rho_{\text {ap }}=$ massa específica aparente $\left(\mathrm{g} . \mathrm{cm}^{-3}\right) ; \mathrm{TU}=$ teor de umidade $(\%) ; \mathrm{W}=$ trabalho absorvido $(\mathrm{J}) ; \mathrm{f}_{\mathrm{bw}}=$ resistência a impacto $\left(\mathrm{KJ} \cdot \mathrm{m}^{-2}\right)$;

$\mathrm{SQ}=$ soma de quadrados; $\mathrm{GL}$ = graus de liberdade; $\mathrm{QM}=$ quadrado médio; $\mathrm{F}=$ valor de $\mathrm{F}$ calculado na linha; ${ }^{\mathrm{ns}}=$ não significativo;

${ }^{*}=$ significativo em $5 \%$ de probabilidade de erro; ${ }^{* *}=$ significativo em $1 \%$ de probabilidade de erro.

climatizada e saturada, respectivamente (Tabela 4). Os resultados encontrados na literatura referentes à massa específica básica ficam entre $0,57 \mathrm{~g} \mathrm{a} 0,59 \mathrm{~g} \cdot \mathrm{cm}^{-3}$ para E. grandis e $0,60 \mathrm{~g}$ a $0,69 \mathrm{~g} \cdot \mathrm{cm}^{-3}$ para E. cloeziana (Ciniglio, 1998; Pereira et al., 2000; Gonçalez et al., 2006; Cademartori et al., 2014).

A massa específica aparente na condição saturada para o E. grandis diferiu estatisticamente das demais condições, apresentando a maior $\rho_{\text {ap }}$. Já para a madeira de E. cloeziana todas as condições de umidade apresentaram diferença significativa entre si, sendo que também na condição saturada a $\rho_{\text {ap }}$ apresentou-se superior. Esse resultado foi similar ao encontrado por Beltrame et al. (2010) que, estudando madeira da espécie Luehea divaricata, obtiveram aumento de
93\% na $\rho_{\text {ap }}$ entre a condição saturada e a climatizada $\left(20{ }^{\circ} \mathrm{C}\right.$ e $65 \%$ de UR). O aumento significativo da $\rho_{\text {ap }}$ na madeira saturada está diretamente relacionado ao crescente aumento de sua massa quando submersa em água. Entretanto a madeira não apresenta o mesmo crescimento volumétrico, que é constante acima do ponto de saturação das fibras (PSF), o que resulta na maior $\rho_{\text {ap }}$.

O trabalho absorvido (W) pelos corpos de prova demonstrou que o E. cloeziana apresentou maior capacidade de absorver e dissipar as cargas de choque comparativamente ao E. grandis. Em relação às condições de umidade, verificou-se que para a madeira de E. cloeziana o aumento no teor de umidade proporcionou um acréscimo significativo no trabalho 
Tabela 4. Valores médios e teste de médias para as propriedades físico-mecânicas da madeira sob influência dos fatores espécie e condição de umidade.

Table 4. Mean values and mean test for both physical and mechanical properties of wood under the influence of the species and moisture condition factors.

\begin{tabular}{|c|c|c|c|c|c|}
\hline Propriedade & Espécie & Seca & Climatizada & Saturada & $\mathbf{F}$ \\
\hline \multirow{5}{*}{$\begin{array}{c}\rho_{\mathrm{ap}} \\
\left(\mathrm{g} \cdot \mathrm{cm}^{-3}\right)\end{array}$} & \multirow{2}{*}{ E. grandis } & $0,49 \mathrm{Aa}$ & $0,51 \mathrm{Aa}$ & $1,07 \mathrm{Ba}$ & \multirow{2}{*}{$1011,53^{* *}$} \\
\hline & & $-0,043$ & $-0,043$ & $-0,051$ & \\
\hline & \multirow{2}{*}{ E. cloeziana } & $0,80 \mathrm{Bb}$ & $0,77 \mathrm{Ab}$ & $1,29 \mathrm{Cb}$ & \multirow{2}{*}{$860,74^{* *}$} \\
\hline & & $-0,049$ & $-0,039$ & $-0,028$ & \\
\hline & $\mathrm{F}^{*}$ & $481,54^{\star *}$ & $441,33^{\star *}$ & $117,52^{\star *}$ & - \\
\hline \multirow{5}{*}{$\begin{array}{l}\text { W } \\
(J)\end{array}$} & \multirow{2}{*}{ E. grandis } & $16,07 \mathrm{a}$ & $15,45 \mathrm{a}$ & $15,54 \mathrm{a}$ & \multirow{2}{*}{$0,05^{\text {ns }}$} \\
\hline & & $-8,11$ & $-5,85$ & $-5,71$ & \\
\hline & \multirow{2}{*}{ E. cloeziana } & $25,16 \mathrm{Ab}$ & $32,32 \mathrm{Bb}$ & $36,74 \mathrm{Cb}$ & \multirow{2}{*}{$21,25^{\star *}$} \\
\hline & & $-4,64$ & $-7,98$ & $-5,06$ & \\
\hline & $\mathrm{F}^{*}$ & $20,69^{* *}$ & $60,84^{* *}$ & $162,4^{* *}$ & - \\
\hline \multirow{5}{*}{$\begin{array}{c}\mathrm{f}_{\mathrm{bw}} \\
\left(\mathrm{KJ} \cdot \mathrm{m}^{-2}\right)\end{array}$} & \multirow{2}{*}{ E. grandis } & $43,10 \mathrm{a}$ & $37,90 \mathrm{a}$ & $40,90 \mathrm{a}$ & \multirow{2}{*}{$0,44^{\mathrm{ns}}$} \\
\hline & & $-21,64$ & $-15,08$ & $-13,45$ & \\
\hline & \multirow{2}{*}{ E. cloeziana } & $69,6 \mathrm{Ab}$ & $82,9 \mathrm{Bb}$ & $86,6 \mathrm{Bb}$ & \multirow{2}{*}{$7,45^{\star \star}$} \\
\hline & & $-12,99$ & $-20,5$ & $-12,21$ & \\
\hline & $\mathrm{F}^{*}$ & $24,11^{* *}$ & $57,91^{* *}$ & $152,9^{\star *}$ & - \\
\hline
\end{tabular}

$\rho_{\mathrm{ap}}=$ massa específica aparente; $\mathrm{W}=$ trabalho absorvido; $\mathrm{f}_{\mathrm{b} w}=$ resistência a impacto; $\mathrm{F}=$ valor de $\mathrm{F}$ calculado na linha (espécie dentro da condição de umidade); $\mathrm{F}^{*}$ = valor de $\mathrm{F}$ calculado na coluna (condição de umidade dentro da espécie); médias seguidas da mesma letra não diferem estatisticamente entre si, minúscula na coluna e maiúscula na linha, em $5 \%$ de probabilidade de erro (Fisher Test, $\mathrm{p}>0,05){ }^{n s}=$ não significativo; ${ }^{* *}=$ significativo em $1 \%$ de probabilidade de erro; valores entre parênteses indicam desvio padrão.

absorvido, demonstrando que a madeira dessa espécie tornou-se mais elástica com o acréscimo de umidade, absorvendo e dissipando melhor as cargas a ela impostas, resultados que seguem a mesma tendência encontrada por outros pesquisadores (Beltrame et al., 2010, 2012). Já para a madeira de E. grandis as condições de umidade não afetaram os valores do trabalho absorvido.

A partir do trabalho absorvido foi possível verificar que ambas as espécies são classificadas como pouco resistentes a choques e quebradiças (Carvalho, 1996). Nesse sentido, a madeira de E. cloeziana e E. grandis não são consideradas aptas para usos que envolvem elevadas cargas de choque. Esse resultado difere do encontrado por Stangerlin et al. (2009) que, estudando as espécies Eucalyptus saligna e Eucalyptus botrioides, obtiveram valor de $\mathrm{W}$ de 4,1 kgm (40,2 J) para ambas as espécies, classificadas como resistente a choques.

Com relação à resistência a impactos, observou-se que E. cloeziana apresentou resultados melhores em comparação com E. grandis. Entre as diferentes condições de umidade não houve diferença significativa $(\mathrm{p}<0,05)$ para a madeira de E. grandis, corroborando resultados apresentados para o W. Esse resultado é similar ao encontrado por Kretschmann (2008) que, estudando a resistência a impactos para de madeira de folhosas saturadas e na condição climatizada $\left(20^{\circ} \mathrm{C}\right.$ e $65 \%$ de UR), não encontrou diferença significativa. Já a madeira de E. cloeziana, $\mathrm{f}_{\mathrm{bw}}$, nas condições saturada e climatizada, foi 24,4\% e 19,1\% superior comparativamente à condição seca. Esses resultados corroboram os obtidos por Beltrame et al. (2010), nos quais a madeira de Luehea divaricata na condição saturada também apresentou-se mais resistente a impactos quando comparada à madeira na condição climatizada $\left(20^{\circ} \mathrm{C}\right.$ e $65 \%$ de UR).

Aanalisando-se os valores do presente estudo, verifica-se que a madeira de E. cloeziana e E. grandis podem serem consideradas como quebradiças e pouco resistentes a choques em todas das condições de umidade, devendo-se evitar a sua utilização em cabos de ferramentas, carrocerias de caminhões e peças em que a madeira é submetida a esforços similares de impacto.

\section{CONCLUSÃO}

A madeira de E. cloeziana foi mais resistente a impactos na condição saturada em comparação com as condições climatizada e seca. Já o E. grandis não apresentou diferença significativa na resistência a impactos nas condições de umidade submetidas. 
Entre as espécies estudadas, o E. cloeziana apresentou maior capacidade de absorver energia e dissipá-la por meio de deformações. Entretanto, as espécies estudadas foram classificadas como pouco resistentes a impactos, não devendo ser utilizadas em locais sujeitos a cargas de choque.

\section{AGRADECIMENTOS}

Os autores agradecem à Coordenação de Aperfeiçoamento de Pessoal de Nível Superior (CAPES) pelo suporte na realização desse trabalho.

\section{STATUS DA SUBMISSÃO}

Recebido: 24 abr., 2014

Aceito: 26 jun., 2016

\section{AUTOR(ES) PARA CORRESPONDÊNCIA}

\section{André Luiz Missio}

Laboratório de Produtos Florestais, Departamento de Ciências Florestais, Universidade Federal de Santa Maria UFSM, Av. Roraima, 1000, Bairro Camobi, CEP 97105-900, Santa Maria, RS, Brasil e-mail: andreluizmissio@gmail.com

\section{REFERENNCIAS}

American Society Testing of Materials - ASTM. D5536-94: standard practice for sampling forest trees for fetermination of clear wood properties. Philadelphia: ASTM; 2010. 9 p. Associação Brasileira de Normas Técnicas - ABNT. NBR7190: projeto de estruturas de madeiras. Rio de Janeiro: ABNT; 1997. 142 p.

Batista DC, Klitzke RJ. Influência do tempo e temperatura de retificação térmica na umidade de equilíbrio da madeira de Eucalyptus grandis Hill ex Maiden. Scientia Forestalis 2010; 38(86): 255-261.

Beltrame R, Gatto DA, Modes KS, Stangerlin DM, Trevisan $\mathrm{R}$, Haselein CR. Resistência ao impacto da madeira de açoita-cavalo em diferentes condições de umidade. Cerne 2010; 16(4): 499-504.
Beltrame R, Mattos BD, Gatto DA, Lazarotto M, Haselein CR, Santini EJ. Resistência ao impacto da madeira de nogueira-pecã em diferentes condições de umidade. Ciência Rural 2012; 42(9): 1583-1587.

Bodig J, Jayne BA. Mechanics of wood and wood composites. 2. ed. Florida: Krieger Publishing Company; 1993. 712 p.

Cademartori PHG, Missio AL, Mattos BD, Schneid E, Gatto DA. Physical and mechanical properties and colour changes of fast-growing Gympie messmate wood subjected to two-step steam-heat treatments. Wood Material Science \& Engineering 2014; 9(1): 1-9.

Carvalho A. Madeiras portuguesas: estrutura anatômica, propriedades, utilizações. São Paulo: Instituto Florestal; 1996. 340 p. vol. VI.

Ciniglio G. Avaliação da madeira serrada de Eucalyptus grandis e Eucalyptus urophylla [dissertação]. São Paulo: Escola Superior de Agricultura Luiz Queiroz, Universidade de São Paulo; 1998.

Echenique MR, Robles FV. Ciencia y tecnología de la Madera I. Veracruz: Universidad Veracruzana; 1993.

Gonçalez JC, Breda LCS, Barros JFM, Macedo DG, Janin G, Costa AF et al. Características tecnológicas das madeiras de Eucalyptus grandis Hill ex Maiden e Eucalyptus cloeziana F. Muell visando ao seu aproveitamento na indústria moveleira. Ciência Florestal 2006; 16(3): 329-341. http:// dx.doi.org/10.5902/198050981912.

Industria Brasileira de Árvores - IBA. Anuário estatístico, ano base 2014. São Paulo: IBA; 2015. 64 p.

Kretschmann DE. The influence of juvenile wood content on shear parallel, compression, and tension perpendicular to grain strength and mode I fracture toughness of loblolly pine at various ring orientation. Forest Products Journal 2008; 58(7-8): 89-96.

Logsdon NB, Calil CJ. Influência da umidade nas propriedades de resistência e rigidez da madeira. Cadernos de Engenharia de Estruturas 2002; 18: 77-107.

Pereira JCD, Pereira JCD, Sturion JA, Higa AL, Higa RCV, Shimizu JY. Características da madeira de algumas espécies de eucalipto plantadas no Brasil. Colombo: Embrapa Florestas; 2000. 113 p.

Stangerlin DM, Melo RR, Santini EJ, Gatto DA, Domingues JMX, Calegari L et al. Avaliação da resistência ao impacto para as madeiras de Eucalyptus botrioides e Eucalyptus saligna. In Anais do Congresso Florestal Estadual; 2008; Nova Palma. Rio Grande do Sul: Congresso Florestal Estadual; 2009. p. 65. 